\title{
BMJ
}

\section{Improving quality of mother-infant relationship and infant attachment in socioeconomically deprived community in South Africa: randomised controlled trial}

${ }^{1}$ School of Psychology, University of Reading, Reading RG6 6AL

${ }^{2}$ Health Systems Research Unit, Medical Research Council, and University of Cape Town, 7505

Tygerberg, South Africa

${ }^{3}$ Department of Psychology,

Stellenbosch University, Matieland 7602, South Africa

${ }^{4}$ Parent Centre and Stellenbosch University, Matieland

${ }^{5}$ Department of Psychiatry and Mental Health, Groote Schuur Hospital, Cape Town, South Africa

${ }^{6}$ Department of Psychiatry, University of Oxford, Oxford OX3 7JX

${ }^{7}$ Department of Obstetrics and Gynaecology, John Radcliffe Hospital, Oxford University, Oxford OX3 9DU

Correspondence to: PJ Cooper p.j.cooper@rdg.ac.uk

Cite this as: BMJ 2009;338:b974 doi:10.1136/bmi.b974

\begin{abstract}
Peter J Cooper, professor in psychopathology, ${ }^{1}$ Mark Tomlinson, specialist scientist, ${ }^{2}$ associate professor, ${ }^{3}$ Leslie Swartz, professor of psychology, ${ }^{3}$ Mireille Landman, clinical psychologist, ${ }^{4}$ Chris Molteno, professor, ${ }^{5}$ Alan Stein, professor of child and adolescent psychiatry, ${ }^{6} \mathrm{Klim}$ McPherson, visiting professor of public health epidemiology, ${ }^{7}$ Lynne Murray, professor in developmental psychopathology ${ }^{1}$
\end{abstract}

\section{ABSTRACT}

Objective To assess the efficacy of an intervention designed to improve the mother-infant relationship and security of infant attachment in a South African peri-urban settlement with marked adverse socioeconomic circumstances.

Design Randomised controlled trial.

Setting Khayelitsha, a peri-urban settlement in South Africa.

Participants 449 pregnant women.

Interventions The intervention was delivered from late pregnancy and for six months postpartum. Women were visited in their homes by previously untrained lay community workers who provided support and guidance in parenting. The purpose of the intervention was to promote sensitive and responsive parenting and secure infant attachment to the mother. Women in the control group received no therapeutic input from the research team.

Main outcome measures Primary outcomes: quality of mother-infant interactions at six and 12 months postpartum; infant attachment security at 18 months. Secondary outcome: maternal depression at six and 12 months.

Results The intervention was associated with significant benefit to the mother-infant relationship. At both six and 12 months, compared with control mothers, mothers in the intervention group were significantly more sensitive (6 months: mean difference $=0.77$ (SD 0.37), $\mathrm{t}=2.10$, $P<0.05, d=0.24 ; 12$ months: mean difference $=0.42(0.18)$, $\mathrm{t}=-2.04, \mathrm{P}<0.05, d=0.26)$ and less intrusive ( 6 months: mean difference $=0.68(0.36), \mathrm{t}=2.28, \mathrm{P}<0.05, d=0.26$; 12 months: mean difference $=-1.76(0.86), \mathrm{t}=2.28$, $\mathrm{P}<0.05, d=0.24)$ in their interactions with their infants. The intervention was also associated with a higher rate of secure infant attachments at 18 months $(116 / 156(74 \%)$ $v 102 / 162(63 \%)$; Wald=4.74, odds ratio=1.70, P<0.05). Although the prevalence of maternal depressive disorder was not significantly reduced, the intervention had a benefit in terms of maternal depressed mood at six months $(z=2.05, P=0.04)$ on the Edinburgh postnatal depression scale).

Conclusions The intervention, delivered by local lay women, had a significant positive impact on the quality of the mother-infant relationship and on security of infant attachment, factors known to predict favourable child development. If these effects persist, and if they are replicated, this intervention holds considerable promise for use in the developing world.

Trial registration Current Controlled Trials ISRCTN25664149.

\section{INTRODUCTION}

Extensive research over the past three decades has shown that when infants experience reliable care that is sensitive to their developmental needs over the first year or so of life, they are likely to develop a secure attachment to their principal carer, usually their mother, ${ }^{1}$ and that this security of attachment endures through to adulthood..$^{2-5}$ In turn, security in the infant's key attachment relationship is associated with better outcomes in several domains of development, in both the short term and the longer term. In early and middle childhood, the benefits of a secure attachment are principally shown in good peer relationships and socioemotional adjustment ${ }^{67}$; in adulthood, associations are consistently found with lower rates of mental health problems. ${ }^{1}$ Notably, emerging evidence also indicates benefits of secure attachments for physical health, including lowered rates of failure to thrive in infancy and early childhood, fewer chronic and recurrent health problems, and better health and lifestyle practices in adulthood (for example, in relation to smoking, drug use, and sexual behaviour), and these benefits are reliably associated with lower health costs. ${ }^{8}$

The capacity of parents to provide the kind of care that promotes security of attachment in infancy and good developmental outcome in childhood can be severely compromised in adverse conditions such as poverty, particularly when mothers have depression..$^{9-11}$ This is of particular concern in 
populations in the developing world, where rates of poverty and of maternal depression after childbirth are high. ${ }^{12}$ Research in Khayelitsha, a peri-urban settlement in South Africa, where a high prevalence of maternal depression was found, also showed, for example, marked impairments in mother-infant interactions compared with those seen in populations in the developed world. ${ }^{13}$ A recent extension to this work showed that these early parenting difficulties were associated with longer term insecurity in the motherinfant attachment relationship. ${ }^{14}$ Interventions in the developed world have been successful in bringing about improvements in mother-infant relationships and infant attachment when they are focused on difficulties in parenting behaviours, ${ }^{15}$ and an urgent need exists to develop such interventions for developing world contexts. Moreover, given the marked limitation in healthcare resources, interventions in the developing world that capitalise on locally available resources are a particular priority.

A small case series in Khayelitsha, in which local mothers from the community were trained to give support to mothers of young infants, produced promising evidence of an improvement in mothers' capacity to interact sensitively with their infants. ${ }^{16}$ The study reported here was a randomised controlled trial of this intervention, which aimed to determine not only whether short term improvements could be brought about in mother-infant interactions but also whether a reduction could be achieved in rates of insecure attachment later in infancy. We had the secondary objective of determining whether the intervention would confer benefits in terms of maternal mood. With regard to

\begin{tabular}{l|l|l|}
\multicolumn{1}{|c|}{ Consecutively identified women $>32$ weeks pregnant $(\mathrm{n}=452)$} \\
\multicolumn{1}{|c|}{ Allocated to intervention and } \\
assessed antenatally $(\mathrm{n}=220)$
\end{tabular}

Flow of participants through study mother-infant interactions, as well as the general characteristic of sensitive responsiveness, we also sought to reduce rates of highly intrusive parenting. We had found that this type of parenting difficulty, in which parents impose their own agenda and cut across, or interfere with, the infant's behaviour, is particularly common and extreme in Khayelitsha; moreover, extensive research has shown that intrusive parenting is a major risk factor for those poor childhood outcomes that are of particular concern in such communities, such as conduct disorder and gang violence. ${ }^{17} 18$ These same childhood behavioural problems are also associated with particular kinds of attachment insecurity - namely, those in which the infant fails to treat the parent as a source of comfort and trust and instead either avoids contact with them when distressed (the avoidant insecure pattern) or else responds in a confused and dysregulated fashion (the disorganised attachment pattern). ${ }^{19}$ When considering attachment outcome, therefore, we were particularly concerned to reduce rates of these two forms of insecurity.

\section{METHOD}

\section{Protocol, design, and objectives}

The study was a randomised controlled trial, in which we randomly assigned pregnant women to an intervention group or a no treatment control group. We made independent assessments, blind to treatment groups, before treatment, at the end of treatment, and at 12 months and 18 months postpartum.

The study took place in two adjoining areas of Khayelitsha (SST and Town II), a peri-urban settlement of between 500000 and 750000 people on the outskirts of Cape Town, South Africa. SST is an informal settlement of shacks characterised by particularly high levels of unemployment and poverty; most shacks are without running water, and considerable overcrowding exists. Many of the inhabitants are recent migrants from rural parts of South Africa. Town II, into which SST merges, is characterised by a somewhat better standard of living. Dwellings are supplied with electricity, most have an indoor water supply, and increasing numbers are served by an indoor waterborne sewage system. Since the election of the ANC government in 1994, a concerted nationwide effort has been made to improve housing conditions; during the study period, much of SST was razed and the inhabitants were accommodated in better housing nearby, similar to that in Town II.

\section{Sample size}

Our own epidemiological work in Khayelitsha showed that most $(58 \%)$ of the mothers were rated as being insensitive to their infant during play interactions (compared with a rate of $25 \%$ in a British sample of non-depressed mothers $\left.{ }^{20}\right) \cdot{ }^{13}$ If the intervention was to effect an improvement by six months in the quality of the mother-infant relationship of at least one third (the same order of improvement as effected by interventions in developed countries ${ }^{1}$ ), this improvement 
Table 1|Characteristics of sample. Values are numbers (percentages) unless stated otherwise

\begin{tabular}{lcc}
$\begin{array}{l}\text { Characteristic } \\
\text { Area: }\end{array}$ & Intervention group $(\mathbf{n}=\mathbf{2 2 0})$ & Control group $(\mathbf{n}=\mathbf{2 2 9})$ \\
\hline SST & $124(56)$ & $121(53)$ \\
\hline Town II & $96(44)$ & $108(47)$ \\
\hline Major depressive disorder & $36(16)$ & $36(16)$ \\
\hline Unplanned pregnancy & $89(40)$ & $83(36)$ \\
\hline Married/cohabiting & $133(60)$ & $140(61)$ \\
\hline First child & $83(38)$ & $83(36)$ \\
\hline Male child & $106(48)$ & $110(48)$ \\
\hline Housing-shack & $185(84)$ & $199(87$ \\
\hline Mean (SD) age (years) & $25.5(5.23)$ & $26.2(5.84)$
\end{tabular}

could be detected ( $\alpha=0.05,80 \%$ power) with two samples of 145 each. A similar degree of improvement in intrusiveness would also be detectable with such samples. (This estimate is conservative, because we intended to analyse the mother-child interaction variables as continuous variables and this would probably mean increased power).

\section{Participants}

Over a period of 22 months, we made efforts to identify and recruit unselected women living in SST and Town II who were in the last trimester of their pregnancy (on the basis of the accounts of their gestation that women had received from the antenatal clinic). Throughout the recruitment period, over three week cycles, a research assistant visited all the homes in both areas to inquire whether anyone had become pregnant or a pregnant woman had moved into the area and to invite identified women to participate in the study.

We anticipated a substantial loss to follow-up, particularly after women had delivered their infants, because many women from rural areas come to the city to use the obstetric services and then return to their rural homes. We identified a consecutive series of 452 women as pregnant within the study area and invited them to take part in the study. Of these, only three refused. After the women had given written informed consent, we made an antenatal assessment to obtain demographic details (such as age and marital status) and information on wider circumstances (such as obstetric factors and housing conditions); we also assessed mental state (see below). We then assigned the 449 women to the intervention or control group by using minimisation, ${ }^{21}$ balancing for antenatal depression, whether or not the pregnancy was planned (that is, factors known to be associated with adverse outcome ${ }^{14}$ ), and whether the mother was living in SST or Town II. We did not pay the women for their participation in the research, but at each assessment we provided a small gift for the infant (an item of clothing). Seven pairs of twins were born, of whom we included only the first born child in the study.

\section{Assignment}

The relevant information from the initial assessment was communicated by telephone to the trial manager
(MT), who ran the minimisation programme and communicated group assignment. The figure shows the progress of participants through the study. By the final assessment at 18 months postpartum, 107 of the 449 women were lost to follow-up, leaving 342 women $(76 \%$ of the original sample); most of the losses occurred, as anticipated, in the six month period after delivery. This is consistent with the known high mobility within this community and equivalent to the proportion lost to follow-up in our previous study in the same two areas. ${ }^{1314}$ The women lost to follow-up did not differ from those retained in terms of demographic characteristics (parity, marital status), apart from age; those lost were somewhat younger than those retained (mean 24.9 (SD 4.4) years versus 26.1 (5.8); $\mathrm{t}=2.07$, $\mathrm{df}=447, \mathrm{P}<0.05)$, and neither did they differ on any of the minimisation criteria or on area of residence.

\section{Intervention}

We based the intervention on an adaptation of a preventive intervention programme by health visitors devised for implementation in Britain, which itself closely follows the principles contained in The Social $B a b y .{ }^{22}$ We adapted this programme for the Khayelitsha project by incorporating the key principles of the World Health Organization's document Improving the Psychosocial Development of Children. ${ }^{23}$ The content and conduct of the intervention was specified in a manual, which was used to train the therapists. (This manual is available at www.reading.ac.uk/psychology/research/ child-development/clinical-subgroup.asp). The aim of the intervention was to encourage the mother in sensitive, responsive interactions with her infant. A major aspect was the use of particular items from the neonatal behavioural assessment schedule ${ }^{24}$ to sensitise the mother to her infant's individual capacities and needs. The intervention was delivered by four women, resident in Khayelitsha, who had been selected with help from the local community council. The women had no formal specialist qualifications, although all were mothers. Two had completed schooling. They received training over a four month period in basic parenting and counselling skills, as well as in the specific mother-infant intervention. An experienced community clinical psychologist (ML) provided them with group supervision throughout the study, on a weekly basis, offering session by session supervision. The intervention was delivered in participants' homes in hour long sessions. The women in the intervention group were visited, ideally, twice antenatally, weekly for the first eight weeks postpartum, fortnightly for a further two months, and then monthly for two months (that is, 16 sessions in total, ending at five months postpartum). Women in the control group received the normal service provided by the local infant clinic (as did the intervention group). This involved fortnightly visits by a community health worker, who assessed the physical and medical progress of mothers and infants, and encouragement of mothers to take their infant to 
Table 2 | Quality of maternal engagement with infant at six and 12 months

\begin{tabular}{|c|c|c|c|c|c|c|}
\hline & \multicolumn{2}{|c|}{ Mean (SD) } & \multicolumn{2}{|c|}{ Difference } & \multirow[b]{2}{*}{$\mathrm{t}$} & \multirow[b]{2}{*}{$P$ value } \\
\hline & $\begin{array}{l}\text { Intervention group } \\
\quad(n=153-9)\end{array}$ & Control group $(n=165-9)$ & Mean (SE) & $95 \% \mathrm{Cl}$ & & \\
\hline \multicolumn{7}{|l|}{ Six months } \\
\hline Sensitivity & $15.35(3.36)$ & $14.58(3.18)$ & $0.77(0.36)$ & 0.048 to 1.492 & 2.10 & 0.037 \\
\hline Intrusiveness & $6.51(2.73)$ & $5.82(2.64)$ & $0.68(0.36)$ & 0.093 to 1.278 & 2.28 & 0.024 \\
\hline \multicolumn{7}{|l|}{12 months } \\
\hline Sensitivity & $5.74(1.88)$ & $5.31(1.51)$ & $0.42(0.18)$ & 0.058 to 0.797 & -2.04 & 0.043 \\
\hline Intrusiveness & $6.41(7.27)$ & $8.17(8.34)$ & $-1.76(0.86)$ & -3.466 to -0.058 & 2.28 & 0.023 \\
\hline
\end{tabular}

the local clinic to be weighed, have their physical health assessed, and be immunised.

\section{Outcomes}

The primary outcomes at six and 12 months were those aspects of the mother-infant relationship previously found to be associated with both socioeconomic adversity and maternal depression (that is, maternal sensitivity and intrusiveness); at 18 months the primary outcome was infant attachment. The secondary outcome was maternal depression (a dichotomous variable for depressive disorder and a continuous variable for depressive symptoms) assessed at six and 12 months.

Assessments took place in a custom-made prefabricated research unit adjoining a local well baby clinic and women's health project, to which the research team brought mothers and infants. The building included a playroom with a one way mirror and an adjoining observation room. Mothers and infants were assessed at six and 12 months postpartum, when mother-infant interactions were observed, and at 18 months postpartum when infant attachment was formally assessed (see below). At six and 12 months postpartum their mental state (see below) was assessed. Mothers and infants in the intervention and controls groups visited the research unit on an equal number of occasions and for equivalent lengths of time, and their experience of the assessment procedures, including the video recording, was similarly equivalent.

\section{Mother-child interactions}

We used age appropriate measures to assess the quality of mother-child interactions at six months and 12 months, by scoring with established, reliable scales. At six months the mothers and infants were filmed in a 10 minute free play interaction, in which we asked mothers to interact with their infants as they would if

Table 3 | Infant attachment categories for intervention and control groups at 12 months. Values are numbers (percentages)

\begin{tabular}{lcc} 
Attachment & Intervention group $(\mathrm{n}=156)$ & Control group $(\mathrm{n}=162)$ \\
Secure & $116(74)$ & $102(63)$ \\
\hline Insecure: & $40(26)$ & $60(37)$ \\
\hline Avoidant & $17(11)$ & $31(19)$ \\
\hline Resistant & $13(8)$ & $13(8)$ \\
\hline Disorganised & $10(6)$ & $16(10)$ \\
\hline
\end{tabular}

they were at home. We rated episodes with the parent/ caregiver involvement scale, which measures the responses of the mother to her infant's needs and initiations..$^{25}$ In view of the literature and our own earlier findings in Khayelitsha, ${ }^{1421}$ our focus was on whether the intervention significantly improved the sensitivity of maternal interactive behaviour and reduced maternal intrusiveness. Accordingly, a trained rater, blind to both treatment group and maternal mental state, scored the videotapes on the five dimensions of "physical involvement," "verbal involvement," "responsiveness," "positive statements," and "control over child's activities," each rated on a five point scale for the "quality" and "appropriateness" of maternal behaviour. We calculated sensitivity as the sum of ratings for the first four dimensions and intrusiveness as the sum of ratings for the last dimension (with codes reversed). We assessed inter-rater reliability on 20 tapes and found it to be uniformly good $(\kappa=0.81$ for sensitivity and $\kappa=0.85$ for intrusiveness).

At 12 months the mother and child were observed in a structured play situation. ${ }^{2627}$ This comprised two three minute episodes, in each of which the mother was asked to play with the infant by using novel toys - a set of stacking rings and a form board. Each typically requires infants of this age to be given support by an adult, so they provided an opportunity to observe how well the mother was able to facilitate the child's play. A trained rater, who was blind to treatment group, maternal mental state, and the interactions at six months, scored maternal behaviour from the videotapes on measures of sensitivity and intrusive-coercive control. We used an established measure to rate sensitivity on a five point scale ${ }^{2627}$; scores were summed across the two tasks. We established intrusive-coercive control by counts of the defined behaviour. ${ }^{27} \mathrm{~A}$ second trained rater, also blind to group and maternal mental state, scored 20 of the videotapes to establish inter-rater reliability: weighted $\kappa=0.91$ for sensitivity, and Pearson $\mathrm{r}=0.83$ for intrusiveness.

\section{Maternal depression}

A trained black South African Xhosa speaking researcher interviewed mothers by using the major depression section of the structured clinical interview for the Diagnostic and Statistical Manual of Mental Disorders, fourth edition (DSM-IV) diagnoses. ${ }^{28}$ The interview had been translated and then back translated 
by following standard principles. ${ }^{29}$ The interviewer and the second author (a clinical psychologist), both of whom were blind to treatment group, reviewed each assessment anonymously, using both tape recordings and the interviewer's notes. A collective decision was made about each of the relevant symptoms, on which the decision about the presence or absence of a DSM-IV major depressive disorder was based. ${ }^{30} \mathrm{We}$ also obtained a continuous measure of depressive symptoms by incorporating the 10 items from the Edinburgh postnatal depression scale, each scored $0-3$, into the structured clinical interview for DSM-IV.

\section{Infant attachment}

At 18 months, we used the strange situation procedure developed by Ainsworth and colleagues to assess infant attachment. ${ }^{31}$ This is a structured, standard procedure, used extensively in research in both the developed and developing world. The assessment has good reliability and predictive validity. ${ }^{32}$ The infant was filmed through a one way mirror, in an unfamiliar playroom over a 21 minute period, during episodes of separation and reunion with the mother and in the presence of a stranger. MT, who had been trained for reliability and was blind to all other information about the infants and their mothers, rated the videotapes. He used the ABCD system ${ }^{313}$; that is, infants were rated as securely attached or insecurely attached, the second of these being specified as avoidant, anxious-resistant, or disorganised. We confirmed reliability by assessing agreement between MT and a second trained rater on 16 tapes $(\kappa=0.96)$.

\section{Statistical analyses}

We used SPSS 12.0 for Windows for descriptive and analytical statistical analyses. We investigated continuous variables for departure from normality by using Shapiro-Wilk's test and compared the treatment groups by means of two sample $t$ tests or Mann-Whitney U tests, as appropriate. For dichotomous outcomes we used $\chi^{2}$ tests to compare groups. We also expressed the differences between the intervention and control groups for the mother-infant interaction variables in terms of effect size by using Cohen's $d^{34}$ As mothers from Town II and SST did not differ on any of the outcomes for either the intervention or the control group, we ignored this variable in the analyses reported below.

Table 4| Maternal depressive disorder and depressive symptoms at six and 12 months for intervention and control group

\begin{tabular}{lcc} 
Depressive disorder-No (\%) & Intervention group & Control group \\
\hline Six months & $21(12.4)(n=170)$ & $29(15.8)(n=184)$ \\
\hline 12 months & $18(10.9)(n=165)$ & $28(15.5)(n=181)$ \\
\hline Depressive symptoms-mean (SD) & $(n=170)$ & $(n=184)$ \\
\hline Six months & $2.78(4.54)$ & $3.91(5.80)$ \\
\hline 12 months & $1.93(4.54)$ & $2.69(5.86)$ \\
\hline
\end{tabular}

\section{RESULTS}

Three quarters of the women in the intervention group received the full 16 sessions, and less than 15\% received fewer than 10 sessions (mean number of sessions $=14.1$, SD 3.92). Table 1 shows the characteristics of the sample. The randomisation process resulted in closely equivalent groups in terms of demographic variables.

\section{Mother-infant interactions}

Table 2 shows scores on the ratings of mother-infant interactions at six months and 12 months. At both time points, compared with mothers in the control group, those in the intervention group were rated as interacting with their children in a significantly more sensitive and less intrusive manner. Cohen $d$ analyses revealed a small effect size for all variables (effect size for sensitivity of $d=0.24$ at six months and $d=0.26$ at 12 months; $d=0.26$ and $d=0.24$ for intrusiveness).

\section{Infant attachment}

Table 3 shows the distribution of classifications of infant attachment. A significantly greater proportion of infants of mothers in the intervention group than infants of control mothers were rated as securely attached at 18 months $(74 \% v 63 \%$; Wald $=4.74$, odds ratio $=1.70, \mathrm{P}=0.029)$. The principal difference between the groups derived from the higher rate of avoidant infants in the control group. The rate of disorganised attachments by infants was also higher in the control group, but not significantly so. We found no association between infant attachment status and the parenting variables. For example, although differing in the expected direction, the mean sensitivity ratings at 12 months for secure versus insecure infants were not significantly different (5.47 $(\mathrm{SD}=1.78)$ and 5.29 (1.52), $\mathrm{t}=0.82, \mathrm{df}=276, \mathrm{P}<0.44)$, and nor were the means for intrusiveness (7.77 (7.97) and 8.00 (8.80), $\mathrm{t}=0.24$, $\mathrm{df}=276, \mathrm{P}<0.82)$.

\section{Maternal depression}

As can be seen in table 4, the prevalence of depressive disorder for the mothers in the intervention group was lower than that in the control group at both assessments; however, the differences were not statistically significant $\chi^{2}=0.85, \mathrm{df}=1, \mathrm{P}=0.36$ at six months; $\chi^{2}=1.16, \mathrm{df}=1, \mathrm{P}=0.21$ at 12 months). With regard to maternal depressive symptoms (the continuous Edinburgh postnatal depression scale measure; see table 4), the mean scores for those in the intervention group were lower at both assessments than were those for the control group, but the benefit of treatment was significant only at six months $(\mathrm{z}=2.05, \mathrm{P}=0.041$ at six months; $\mathrm{z}=0.24, \mathrm{P}=0.813$ at 12 months). Neither maternal depressive disorder nor maternal depressive symptoms were correlated with the parenting variables or infant attachment. For example, at six months postpartum the correlations between the Edinburgh postnatal depression scale score and sensitivity was $\mathrm{r}=0.04$, and that between the depression scale score and 


\section{WHAT IS ALREADY KNOWN ON THIS TOPIC}

Mother-infant interactions are adversely affected by both socioeconomic adversity and maternal depression

Such impaired interactions are associated with adverse psychological, social, and physical consequences for child development

Some evidence from Western countries shows that interventions directed at mother-child interactions can improve mother-child interactions and childhood outcome

\section{WHAT THIS STUDY ADDS}

A home based mother-infant intervention, delivered by trained lay therapists in the context of socioeconomic disadvantage, has positive benefits for both maternal responsiveness and children's emotional development

This low cost approach could be integrated within health systems at a population level to deliver interventions to mothers and their infants in the developing world

intrusiveness was $\mathrm{r}=0.10$; the mean Edinburgh postnatal depression scale scores for secure (3.61, SD 5.34) and insecure $(3.35,5.59)$ infants were similar $(\mathrm{t}=0.37$, $\mathrm{df}=286, \mathrm{P}<0.71)$.

\section{DISCUSSION}

Compared with women who received no specific help or support, women in Khayelitsha who received a home based intervention interacted with their infants with greater sensitivity and with less intrusiveness, both shortly after completion of the intervention (six months postpartum) and at a longer term follow-up (12 months postpartum). The magnitude of improvement in parenting was of the same order as reported in studies in the developed world of supportive interventions that focused on sensitivity. ${ }^{15}$ At 18 months postpartum infants whose mothers had received the intervention were more likely to be rated as securely attached to their mothers than were infants of control group mothers. Given the reliable relation between early insecure attachment and later childhood behavioural problems, the fact that more than $10 \%$ fewer of those who received treatment were insecure compared with the controls means that early intervention could have the potential to make an important contribution to reducing the rate of subsequent childhood disturbance.

Although we found no difference between treated and untreated mothers in terms of the prevalence of depressive disorder, the intervention had some benefit in terms of maternal mood at six months (as measured by the Edinburgh postnatal depression scale). The limited effect of the intervention on maternal depression is consistent with other research showing that interventions principally directed at the mother-infant relationship do not necessarily bring about improvements in maternal mood. ${ }^{35}$ Prevalence of depressive disorder in both the control and the intervention group in this study were somewhat lower than those found in our previous epidemiological study, possibly reflecting the steady improvement in living conditions in the intervening period, which may have alleviated a key source of distress in the women's lives.
The positive impact of the intervention on infant attachment is in line with the findings of previous research on the benefit of early mother-infant interventions, although, to our knowledge, this is the first demonstration of such a benefit in a developing world context. The effect size for maternal sensitivity is similar to the difference in sensitivity between depressed and non-depressed postpartum women living in conditions of adversity in the United Kingdom, ${ }^{1320}$ a magnitude of difference that strongly predicts adverse cognitive development. ${ }^{36}$ The absence of an association in our study between security of infant attachment and improvement in the specific features of the mother-infant interactions measured is not inconsistent with the previous literature ${ }^{37}$; the intervention may have improved dimensions of parenting related to attachment that were not assessed in our study. Although the benefit of the intervention could have been merely a function of the women in the intervention group receiving more attention than women in the control group, this is unlikely as two recent reviews of early intervention studies have concluded that attention not directed specifically at mother-infant engagement has no impact on the quality of the mother-child relationship. ${ }^{3538}$

The findings of this study show that the benefits to the mother-infant relationship of an early intervention in developed world contexts similarly obtain in the deprived peri-urban conditions of Khayelitsha. The intervention was delivered by women from the local community who had no formal training, apart from that received from the study team for delivery of the intervention. In addition, they had a focused task (rather than responsibility for comprehensive community health), they were given appropriate support and supervision, and they had strong community support, all of which are regarded as essential for effective community health worker programmes. ${ }^{39}$ This indicates that the intervention is potentially sustainable and that it could be "scaled up" in developing countries with relatively limited resources. Clearly, the design of the study precludes any discussion of whether similar, or superior, outcomes might have been achieved if a different form of intervention had been delivered.

\section{Strengths and weaknesses}

Despite the difficult developing world circumstances, we were nevertheless able to do a randomised trial of a mother-infant intervention in which rigorous trial procedures were followed: a standardised psychological intervention was delivered, and systematic independent assessments were made. Indeed, "gold standard" assessments were made of both the mother-infant relationship and outcomes in infants. We were able to train lay community workers to deliver the intervention to a high standard, and the intervention itself, conceived in a developed world context, was highly acceptable to the women receiving it, with very low dropout rates for those who did not move away. That we could not 
investigate which aspects of the treatment were particularly effective was unfortunate.

\section{Questions and future research}

Our findings raise two important questions. Firstly, determining whether the improvements in the early mother-infant interactions and infant attachment are realised in subsequent positive developmental outcomes in children is important. The children living in areas such as Khayelitsha are at considerable risk of poor physical and emotional health, violence, educational failure, and a host of other associated adverse outcomes. ${ }^{40}$ If the improvements in mother-infant interactions and infant attachment were shown to be of benefit in terms of these long term outcomes, this would be very important. The fact that rates of avoidant and disorganised attachment were especially low for infants in the intervention group is particularly encouraging, as these are forms of insecure attachment associated with subsequent conduct problems in children. ${ }^{41}$ Secondly, the positive results produced in this study arose in the context of a tightly delivered research intervention. That the intervention can be developed into a sustainable and widely available service that produces benefits similar to those obtained in this study needs to be shown. The fact that such positive outcomes were obtained by using lay therapists is particularly encouraging in this regard. This suggests that the intervention is not only sustainable but has the potential to be scaled up at low cost in resource constrained settings in developing countries.

We are grateful to the Parent Centre, a Cape Town based nongovernmental organisation, for providing the infrastructure for the recruitment and training of the community workers. We thank Marjorie Feni, Nomabili Siko, Nokwanda Sikana, and Lephina Makhanya, the community workers in this study; Nosisana Nama and Busisiwe Magaze who assisted in the assessment of the mothers and infants; Thulani Njengele and Agnese Fiori-Cowley for coding of the video recordings; and Timothy Freeth for help with data entry and general administration. We are especially indebted to the mothers and children who took part in this study.

Contributors: PJC designed the study and supervised its overall conduct, managed the data analysis, and took the lead in writing the paper. MT was the trial manager and trained and supervised the assessors. LS assisted in training the assessors. ML trained and supervised the community workers and assisted in drafting the treatment manual. CM assisted in setting up recruitment procedures. AS assisted in the development of the intervention and the overall supervision of the study. KMacP supervised the data analysis. LM developed the intervention manual and assisted in training the community workers, doing the assessments, and training the assessors. All authors took an active role in writing the submitted paper and approved the final version. PJC is the guarantor

Funding: This study was supported by a grant (B574100) from the Wellcome Trust. MT was supported by a fellowship from the Vlotman Trust.

Competing interests: None declared

Ethical approval: The research ethics committees of the University of Reading and the Health Sciences Faculty of the Medical School of the University of Cape Town approved this study.

1 De Wolff MS, van ljzendoorn MH. Sensitivity and attachment: a metaanalysis on parental antecedents of infant attachment. Child Dev 1997;68:571-91.

2 Waters E, Merrick S, Treboux D, Crowell J, Albersheim L. Attachment security in infancy and early adulthood: a twenty-year longitudinal study. Child Dev 2000;71:684-9.

3 Hamilton CE. Continuity and discontinuity of attachment from infancy through adolescence. Child Dev 2000;71:690-4.
4 Sroufe LA, Egeland B, Carlson E, Collins WA. Placing early attachment experiences in developmental context: the Minnesota longitudinal study. In: Grossmann KE, Grossmann K, Waters E, eds. Attachment from infancy to adulthood. New York: Guilford Press, 2005.

5 Main M, Hesse E, Kaplan N. Predictability of attachment behavior and representational processes at 1,6, and 19 years of age: the Berkeley longitudinal study. In: Grossmann KE, Grossmann K, Waters E, eds. Attachment from infancy to adulthood. New York: Guilford Press, 2005.

6 Greenberg MT. Attachment and psychopathology in childhood. In: Cassidy J, Shaver PR, eds. Handbook of attachment. New York: Guilford Press, 1999.

7 Schneider B, Atkinson L, Tardiff C. Child-parent attachment and children's peer relations: a quantitative review. Dev Psychol 2001;37:86-100.

8 Ranson KE, Urichuk LI. The effect of parent-child attachment relationships on child biopsychosocial outcomes: a review. Early Child Dev Care 2008;178:129-52.

9 Martins C, Gaffan EA. Effects of early maternal depression on patterns of infant-mother attachment: a meta-analytic investigation. / Child Psychol Psychiatry 2000;41:737-46.

10 Atkinson L, Paglia A, Coolbear J, Niccols A, Parker KCH, Guger S. Attachment security: a meta-analysis of maternal mental health correlates. Clin Psychol Rev 2000;20:1019-40.

11 Campbell SB, Brownell CA, Hungerford A, Speiker SJ, Mohan R, Blessing JS. The course of maternal depressive symptoms and maternal sensitivity as predictors of attachment security at 36 months. Dev Psychopathol 2004;16:231-52.

12 Patel V, Rodrigues M, DeSouza N. Gender, poverty and postnatal depression: a study of mothers in Goa, India. Am J Psychiatry 2002;159:43-7

13 Cooper PJ, Tomlinson M, Swartz L, Woolgar M, Murray L, Molteno C. Postpartum depression and the mother-infant relationship in a South African peri-urban settlement. Br J Psychiatry 1999;175:554-8.

14 Tomlinson M, Cooper PJ, Murray L. The mother-infant relationship and infant attachment in a South African peri-urban settlement. Child Dev 2005;76:1044-54.

15 Bakermans-Kranenburg MJ, van Izendoorn MH, Juffer F. Less is more: meta-analyses of sensitivity and attachment interventions in early childhood. Psychol Bull 2003;129:195-215.

16 Cooper P, Landman M, Tomlinson M, Molteno C, Swartz L, Murray L. The impact of a mother-infant intervention in an indigent peri-urban South African context: pilot study. Br J Psychiatry 2002;180:76-81.

17 Granic I, Patterson GR. Towards a comprehensive model of antisocial behavior: a dynamic systems approach. Psychol Rev 2006;113:101-31.

18 Patterson GR. Coercive family process. Eugene, OR: Castalia Press, 1982.

19 Solomon J, George C. The measurement of attachment security in infancy and childhood. In: Cassidy J, Shaver PR, eds. Handbook of attachment: theory, research and clinical applications. New York: Guilford Press, 1999:287-316.

20 Murray L, Fiori-Cowley A, Hooper R, Cooper P. The impact of postnatal depression and associated adversity on early mother-infant interactions and later outcome. Child Dev 1996;67:2512-26.

21 Pocock SJ, Simon R. Sequential treatment assignment with balancing for prognostic factors in the controlled clinical trial. Biometrics 1975;31:103-15.

22 Murray L, Andrews E. The social baby. London: The Children's Project, 2002.

23 World Health Organization. Improving the psychosocial development of children. Geneva: WHO, 1995.

24 Brazelton TB, Nugent JK. Neonatal behavioral assessment scale: clinics in developmental medicine. London: McKeith Press, 1995.

25 Farran DC, Kasari K, Comfort M, Jay S. Parent/caregiver involvement scale. Nashville, TN: Vanderbilt University, 1986.

26 Wolke D, Skuse D, Mathison B. Behavioural style in failure to thrive infants: a preliminary communication. J Pediatr Psychol 1990;15:237-53.

27 Stein A, Wooley H, Cooper SD, Fairburn CG. An observation study of mothers with eating disorders and their infants. J Child Psychol Psychiatry 1994:35:733-48.

28 First MB, Spitzer RL, Gibbon M, Williams JB. Structured clinical interview for DSM-IV axis 1. New York: New York State Psychiatric Institute, 1996.

29 Brislin RW. The wording and translation of research instruments. In: Lonner WJ, Berry JW, eds. Field methods in cross-cultural research. London: Sage, 1986.

30 American Psychiatric Association. Diagnostic and statistical manual of mental disorders. 3rd ed. Washington, DC: American Psychiatric Association, 1980.

31 Ainsworth MDS, Blehar M, Waters E, Wall S. Patterns of attachment: a psychological study of the strange situation. New Jersey: Lawrence Erlbaum, 1978. 
32 Teti DM, Nakagawa M. Assessing attachment in infancy: the strange situation and alternate systems. In: Gibbs E, Teti DM, eds. Interdisciplinary assessment of infants: a guide for early intervention professionals. Baltimore: Paul H Brookes, 1990:191-214.

33 Main M, Solomon J. Procedures for identifying infants as disorganized/disoriented during the Ainsworth strange situation. In Greenberg MY, Cicchetti D, Cummings EM, eds. Attachment in the preschool years: theory, research, and intervention. Chicago: University of Chicago Press, 1990:35.

34 Thalheimer W, Cook S. How to calculate effect sizes from published research articles: a simplified methodology. 2002. http://worklearning.com/effect sizes.htm.

35 Nylen K, Moran T, Franklin C, O’Hara M. Maternal depression: a review of relevant treatment approaches for mothers and infants. Infant Ment Health J 2006;27:327-43.

36 Murray L, Hipwell A, Hooper R, Stein A, Cooper PJ. The cognitive development of five year old children of postnatally depressed mothers. J Child Psychol Psychiatry 1996;37:927-35.
37 Van ljzendoorn MH, Juffer F, Duyvesteyn MGC. Breaking the intergenerational cycle of insecure attachment: a review of the effect of attachment based interventions on maternal sensitivity and infant security. J Child Psychol Psychiatry 1995;36:225-48.

38 Murray L, Halligan SL, Cooper PJ. Effects of postnatal depression on mother-infant interactions, and child development. In: Wachs T, Bremner G, eds. Handbook of infant development. Oxford: WileyBlackwell (in press).

39 Haines A, Sanders D, Lehmann U, Rowe AK, Lawn JE, et al. Achieving child survival goals: potential contribution of community health workers. Lancet 2007;369:2121-31.

40 Barbarin 0, Richter LM. Mandela's children: growing up in postapartheid South Africa. London: Routledge, 2001.

41 Belsky J, Fearon RMP. Infant-mother attachment security, contextual risk, and early development: a moderational analysis. Dev Psychopathol 2002;14:293-310.

Accepted: 9 December 2008 